\title{
Mujer y nación en el «discurso» de la intelligentzia en el amanecer de las repúblicas. Un análisis comparativo entre Colombia y Argentina
}

\section{Nathalie Goldwaser}

\section{(2) OpenEdition Journals \\ Edición electrónica \\ URL: http://journals.openedition.org/bifea/1525 \\ DOI: $10.4000 /$ bifea. 1525 \\ ISSN: 2076-5827 \\ Editor \\ Institut Français d'Études Andines}

\section{Edición impresa}

Fecha de publicación: 1 agosto 2011

Paginación: 399-409

ISSN: 0303-7495

\section{Referencia electrónica}

Nathalie Goldwaser, « Mujer y nación en el «discurso» de la intelligentzia en el amanecer de las repúblicas. Un análisis comparativo entre Colombia y Argentina », Bulletin de l'Institut français d'études andines [En línea], 40 (2) | 2011, Publicado el 01 febrero 2012, consultado el 07 noviembre 2020. URL http://journals.openedition.org/bifea/1525; DOI : https://doi.org/10.4000/bifea.1525

\section{(c)}

Les contenus du Bulletin de l'Institut français d'études andines sont mis à disposition selon les termes de la licence Creative Commons Attribution - Pas d'Utilisation Commerciale - Pas de Modification 4.0 International. 


\title{
Mujer y nación en el «discurso» de la intelligentzia en el amanecer de las repúblicas. Un análisis comparativo entre Colombia y Argentina
}

\author{
Nathalie Goldwaser*
}

\section{INTRODUCCIÓN}

Las investigaciones comparativas son un medio particularmente eficaz, pero muy poco practicado, para analizar las variaciones nacionales, ya que permitirían saber si los fenómenos comparables son producto de iguales estructuras o de la circulación de modelos culturales (Helibron, 2009). La respuesta nunca es a priori, solamente a posteriori y sobre la base de un análisis empírico:

«La historia comparada es capaz de revelarnos interacciones antes no conocidas entre las sociedades (...) La comparación podrá revelar, entre sociedades históricamente diferentes, relaciones extremadamente antiguas de las que (...) no se podría señalar filiación común» (Bloch, 1992: 86).

Según Víctor Uribe-Uran (2006), el proceso revolucionario de independencia de la Nueva Granada se asemeja al proceso del distante Río de la Plata. En ambas naciones, las actividades de la elite fueron consistentemente más intensas e influyentes en la marcha del país.

Politóloga, Magister en Comunicación y Cultura de la Universidad de Buenos Aires (UBA), becaria doctoral CONICET - Inst. de Inv. Gino Germani (IIGG-FSOC). Investigadora asociada al Instituto Francés de Estudios Andinos (IFEA UMIFRE 17, CNRS-MAEE). Docente de la materia Historia del Arte de la Facultad de Arquitectura, Diseño y Urbanismo (UBA). E-mail: nathalie.goldwaser@gmail. com 
Una de esas consecuencias, sin lugar a dudas, ha sido la adopción por parte de los hombres opositores al régimen de la colonia española, de una filosofía política innovadora para hacer frente a la separación de la metrópolis. La irrupción del liberalismo y del romanticismo europeo (fundamentalmente de España, Inglaterra y Francia) en las ideas tanto de los integrantes de la llamada «Generación del '37» argentina1 como en los hombres del grupo del general Francisco de Paula de Santander2, dieron herramientas para la emergencia de los conceptos de ciudadanía-individuo-nación. Tanto un grupo como otro recurren, entre otros géneros, a la novela histórica como estrategia para consolidar aquellas ideas. Por ejemplo, María Dolores o la historia de mi casamiento (1841) de José Joaquín Ortiz; Rosina o la prisión del castillo de Chágres (1842) e Ingermina o la Hija del Calamar (1844) de Juan José Nieto Gil (pertenecientes a la Nueva Granada); «La cautiva» de Esteban Echeverría, aparecida en el Río de la Plata en 1837 bajo el título Rimas y una de las más célebres obras de este poeta-padre fundador de la «Generación del '37», en donde logró incorporarle alcance social ya que reflejó, desde las primeras páginas, el conflicto de su tiempo: la convivencia de la barbarie con la «civilización-por-venir». Como se habrá advertido, en los tres se encuentra la presencia de «una mujer» en el título de la obra.

Si partimos de considerar que la literatura es subsidiaria de la política como la política de la literatura, por lo tanto mucho tendrá que ver el análisis de estas obras con respecto a la formación de la nación en ambos países. No obstante, en el presente avance de investigación se propone exponer un «estado del arte» pesquisando todos aquellos trabajos contemporáneos que han advertido (o no) de la presencia de la figura de la mujer en la narrativa «política» de la primera mitad del siglo XIX.

Se considera de fundamental importancia para la cultura en formación, el desarrollo de una literatura que no solo se diferencie de los elementos de la época colonial (cuyo estilo característico era el desenvolvimiento de la crónica, memoriales de conquistas militares, hagiografías, poemas epopéyicos, etc.), sino también que siente las bases para un espíritu moderno. En este sentido, el Estado-nación constituye el cuadro de referencia privilegiado para la identidad de los individuos en la modernidad, marcando profundamente sus hábitos sociales a través de la educación y la promoción de un espíritu de pertenencia a la comunidad nacional nutrido de rasgos que se instituyen hegemónicamente, tales como la lengua, la historia compartida a través de las leyendas, los mitos, la música y otros bienes culturales. Dentro de estos, la lengua y la literatura resultan fundamentales en

1 Fundamentalmente consideramos a Esteban Echeverría, Juan Bautista Alberdi, Juan María Gutiérrez, Vicente Fidel López y Domingo Faustino Sarmiento.

2 En particular, nos referimos a un grupo políticamente heterogéneo —reúne tanto a liberales como a conservadores - y está compuesto por: Juan José Nieto Gil, Florentino González, José Joaquín Ortiz, Juan Francisco Ortiz, Eladio Vergara, José Antonio de Plaza, José María Ángel Gaitán, Felipe Pérez, Raimundo Bernal Orjuela y Eugenio Díaz. Casi todas estas figuras participaron activamente, como intelectuales y como políticos, en el debate que intentaba definir el mapa político y la identidad cultural de la naciente república (véase González, 2006). 
la producción de representaciones colectivas (Jurt, 2009). De ahí la necesidad de fijar la vista en los intelectuales, literatos, publicistas y pensadores del mundo social; ya sea como anunciadores de un futuro radiante, nostálgicos de un pasado lejano o profetas del presente. El mundo intelectual en los albores de la nación se presenta no como un espacio desencarnado, desplegado exclusivamente en el cielo de las ideas, sino como un universo social formado por agentes, individuos e instituciones que producen significaciones dignas de análisis.

Ingermina o la novia del calamar, la primera novela histórica colombiana, fue producida inmediatamente después de la independencia de la Nueva Granada. En la trama de dicha obra se produce un encuentro amoroso entre una princesa india y un colonizador español, visto desde los ojos de un autor exiliado como lo fue Juan José Nieto. Lo verdaderamente sorprendente es que hay un desinterés tanto por esta obra como, en general, por el papel del autor en la escena nacional. Es frecuentemente extraña la incorporación de dicha obra en las escuelas. Algunos afirman que se debe a motivos racistas: fue el único presidente negro y, por ende, hubo una manipulación histórica y una invisibilización de su presencia en el mundo literario y político3; otros, porque María de Jorge Isaacs ganó la escena hegemónica literaria por referirse precisamente a la función de la Iglesia en Colombia (obra que además es leída hoy incluso en las escuelas argentinas). Por ello, nace el interrogante: ipor qué, si Ingermina ha sido una «antesala» a la obra de Isaacs, es mayormente ignorada?

Ingermina... es obra de la pluma de un nuevo-granadino que fue encarcelado y luego exiliado. Allí, es posible rastrear concepciones y conceptos en relación a la mujer y la conformación de la idea de Patria o Nación. Solo por dar algún ejemplo: Ingermina, la joven «india», bella, de rasgos atractivos para los españoles, hija del cacique máximo de la colonia de Calamar, es colmada de atenciones por Alonso Heredia, hermano del gobernador-colonizador. La india, con la amorosa instrucción de aquel, adopta la religión cristiana, además de haber aprendido a leer, escribir y expresarse como «mujer civilizada». Para Alonso, Ingermina se convirtió en objeto de deseo y posesión porque «la joven Calamareña descendía de los soberanos de la Tierra. Orgullo propio de casi todo español, que siempre quiere ser hijo de algo» — dice el narrador- aceptando que era necesario «enseñarle a hablar español, poniendo además gran empeño en que fuese poco a poco abandonando los hábitos nacionales» (Nieto Gil, 2001 [1844]: 66).

El autor en un párrafo condensa las características de los sujetos de ambos continentes. Y es una mujer la que media entre un lado y el otro. Ingermina logra «civilizarse» sin nunca abandonar sus principios territoriales, nacionales - como lo sugiere el autor-.

Siguiendo el trabajo de María Inés de Torres (1995), si se compara el «discurso nacional» y el «discurso amoroso» (la ficción), puede corroborarse que la creación del marco jurídico y geográfico de una naciente nación no es el acontecimiento

3 Véase «Colombia borró de la historia a su único presidente negro» (Gonzálo Guillen, El nuevo Herald, 12 de nov. de 2008). 
decisivo. Se puede obedecer a la constitución de una Nación aunque no por eso se la quiere, se la desea. Y quizás esté allí la respuesta a por qué Benedict Anderson afirma que la nación es un «artefacto cultural» cuya definición es la de ser una comunidad imaginada.

Considerar al «discurso amoroso» como antesala al discurso nacional puede arrojar claves para comprender la construcción de la idea de Nación tanto en el Río de la Plata como en la Nueva Granada. Tal como sucede con el discurso nacional, el discurso amoroso también tiende a «convertirse en un dispositivo de disciplinamiento». Pero esta similitud tiene su asimetría: el discurso nacional se nutriría del discurso amoroso. El primero, para disciplinar y legitimar el nacionalismo a través del sentimiento (el ejemplo más claro es el patriotismo que se presenta como una fuerza que «emana» o debiera emanar naturalmente del corazón de todo ser humano, sino se es un apátrida o antipatriota). El segundo, el amoroso, para regular las relaciones entre los sexos. En ambos casos, la fidelidad es el punto capital: "sólo se tiene una patria, la de origen, y sólo se ama a una persona», por eso el Otro, el tercero, siempre es presentado como un obstáculo o amenaza. El vínculo entre ambos discursos produce una forma concreta, un tipo de «discurso sobre el género»; es decir, sobre lo masculino y lo femenino. Lo interesante de esta perspectiva es que se constata que dicho análisis puede aplicarse parcial o totalmente a los discursos de otros países de Latinoamérica y, por qué no, del resto del mundo occidental.

Se explica, de este modo, por qué buena parte de la narrativa patriótica está compuesta por historias de amor — con ciertos rasgos de erotismo— que luego se convertirán en historias de amor frustradas o fracasadas.

El interés del siglo XIX europeo por la mujer como objeto de preocupación científica (y artística) tenía por causa, principalmente, la alteración que significó la «Revolución Industrial» en la vida familiar. Bien se sabe que en el amanecer de las repúblicas latinoamericanas no hubo un acontecimiento de tales características, por tanto, en el momento en que se estaba diseñando el «mapa de la nueva nación», la ideología patriarcal aparece como un fuerte elemento civilizador: La mujer «bárbara», no sometida todavía a las leyes del decoro y las buenas costumbres, es vista como una amenaza al orden que otorgara la familia. Dicho orden, repetimos, es considerado como la piedra angular del Estado-nación. Por tanto, la misión de la mujer «disciplinada» debe ser la de formar a los nuevos ciudadanos, la de «imbuirlos» en el espíritu nacional a través de lo doméstico, sin interferir en la vida pública.

A pesar de que el nacionalismo debía suceder en el ámbito «real», el de la política y el erotismo, solo en los relatos literarios, y escondido en los fueros íntimos, la imagen privilegiada por el discurso del romanticismo liberal es su concepción de la historia de la patria como una historia difícil de amor, aunque inevitable para vislumbrar un destino. Ninguna de las narraciones de amor tiene un final feliz, sobre todo para los personajes femeninos. A su vez, fomentan el maniqueísmo de aquella estética romántica: el enfrentamiento civilización-barbarie donde es posible interpretar que uno debe exterminar al otro: no hay posibilidad de convivencia. 
Estas retóricas ideológicas que retroalimentan el universo simbólico, son «dos caras del mismo proyecto civilizador». Ocuparse de la mujer es solo el modo de garantizar el orden de la ideología patriarcal. Por lo tanto, se desexualiza a la «mujer civilizada»; mientras que a aquellas mujeres que ponen en peligro su plan de nación, se les coloca actitudes salvajes que terminan por confundir su sexo.

El binomio civilización-barbarie se reactualiza y no agota el análisis. Asimismo, el exilio pareciera cumplir un rol fundamental en estas obras: en el caso de la Nueva Granada, éste estuvo provocado por lo que se denominó «Guerra de los Supremos» (1839-1841). Conflicto que tuvo por causa el intento de consolidar la dictadura del general Urdaneta frente a sus opositores (el grupo del general Santander). El problema central era «la definición de quiénes son los «verdaderos patriotas〉 con derecho a participar plenamente en la vida política nacional: la resistencia de los partidarios de Santander a la presencia de los partidarios de esas dictaduras en la burocracia nacional y regional se constituyó en el trasfondo nacional de esa guerra civil» (González, 2006: 402); en el caso de los miembros de la «Generación del '37» del Río de la Plata, también debieron exiliarse por el régimen de Rosas. Estos elementos permiten reconstruir una cartografía común entre ambos países.

\section{ACLARACIONES METODOLÓGICAS}

La figura de la mujer tanto en Colombia como en Argentina se convierte en una arista original para poder reconstruir un aspecto de la historia política del concepto Nación, y a la vez permite rastrear cuáles son las herencias que el imaginario de la época nos legó. El problema de investigación es planteado en los siguientes términos: en aquellos momentos donde el mencionado concepto se pone en juego dentro de los discursos y proyectos de las elites intelectuales, bajo el signo de una aspiración universalista, invocar a la figura de la mujer podría interpretarse como una contradicción. Sin embargo, y dado que no se han planteado ese tema, se cuestiona por el imaginario alrededor de la figura «mujen» por la cual se incluye dentro del discurso y, al mismo tiempo, se excluye de la representación dentro de la esfera política.

Se ha escogido el concepto figura para hacer referencia a la «mujer escrita» porque justamente da cuenta de un «objeto» aún no contenido en un status o marco jurídico-político, como lo puede ser el status de ciudadanía. Además, permite separar en relación con la experiencia de un sujeto particular. Hablar de «figura» es referirse a una imagen, una idea o una unidad ya asignada o puesta en relación a un concepto-clave.

Hemos construido una tipología que permite clasificar el tratamiento dado a la mujer tanto por parte de la literatura perteneciente a la elite intelectual decimonónica, como por los estudios contemporáneos de ambos países, a saber:

1) La que se refiere a ella como una excusa, un pretexto; esto es, herramienta (arma) discursiva para poder apelar a cuestiones de otro tenor, sobre todo en tiempos de censura o no libertad de expresión; 
2) La figura de la mujer cual objeto (de ilustración); es decir, como destinataria predilecta, como ideal de «público» para recepcionar las producciones discursivas de los hombres románticos e ilustrados. La «mujer objeto» ejemplifica un ideal: el de un receptor que escucha, lee pero no decide ni critica, es el ideal de un público que se está formando para la república, el componente primordial para el orden social y para el acatamiento de la política de la época que es la que abriría paso discursivamente (pero no sólo) a la legitimidad de un Estado;

3) Por último, la figura de la mujer puede convertirse en una intrusa, un obstáculo para el progreso. Una figura que rompería con la fraternidad y armonía entre los iguales, los hombres4.

Estos tres tipos no encarnan a una o a un grupo de mujeres, sino que son modos de presentar en escena a la mujer, permitiendo dibujar los distintos imaginarios de la época.

Por último, revisando las publicaciones consultadas se puede apreciar que existe una tendencia generalizada. Por un lado, los estudios actuales, vistos en su conjunto, que refieren a la mención o no mención de la mujer en los escritos políticos de comienzos del siglo XIX, no problematizan los conceptos políticos de aquellos discursos —nación, ciudadanía, república, etc.—5. Por el otro, los que efectivamente colocan a la política y a los conceptos políticos en el centro de sus escritos para referirse a la historia, olvidan — la mayoría de las veces — la incorporación o función de la mujer en el «contexto de los textos» fundacionales. Por ello, el objetivo es poder articular ambas tendencias. Se propone estudiar la historia de la construcción del concepto nación y ciudadanía desde el presente hacia el pasado. Es decir, registrar en las fuentes secundarias si a la hora de analizar los conceptos políticos, toman o no en cuenta a la mujer (sea como sujeto que actúa en el «mundo de la vida» o como «mujer escrita», figurada). Tal como lo propondría Marc Bloch, un conocimiento histórico requiere una historia retrospectiva donde construyendo cuadros sucesivos desde el presente hacia el pasado, el historiador sea capaz de proyectar la película hacia atrás.

\section{INVESTIGACIONES HISTÓRICAS SOBRE LA MUJER, EXPLICACIONES AUSENTES SOBRE LA NACIÓN}

Una amplia bibliografía acerca del siglo XIX acuerda que a la mujer se la ha invisibilizado en la historia tanto de Argentina como de Colombia. Han tratado

4 Ésta es una figura inspirada en La intrusa de Borges cuyo personaje, Juliana Burgos, es a la vez la cautiva de los hermanos Nilsen, indecible y siniestra mujer que viene a romper la calma entre los hermanos y a producir una tragedia que deviene en guerra y muerte. Aquella mujer había empezado a ser sujeto de deseo, y ante la imposibilidad de un acuerdo entre los Nilsen (o administración de la cosa en disputa) se resuelve, parafraseando a Borges, por la eliminación monstruosa.

5 Tal es el caso, por ejemplo, de las Actas de la IX Cátedra Anual de Historia, «Ernesto Restrepo Tirado» (Bogotá, 2004) cuya temática ha sido Mujer, nación identidad y ciudadanía: siglos XIX y XX. A lo largo de los artículos de este libro se ha intentado rastrear definiciones en torno a los conceptos mencionados anteriormente sin obtener resultados significativos que los problematicen. 
esta cuestión los siguientes autores: para el primer caso Francine Masiello, María Inés de Torres, Graciela Batticuore, Dora Barrancos, María Gabriela Mizraje, José Emilio Burucúa, Donna Guy, Fernanda Gil Lozano, María Gabriela Ini, Valeria Silvina Pita y Mercedes Sacchi, entre otros; para el caso colombiano se puede mencionar a Suzy Bermúdez, Evelyn Cherpak, Guiomar Dueñas Vargas y Magdala Velásquez Toro.

En este avance de investigación nos detendremos en tres de estos estudios sobre la construcción de la Nación en La Nueva Granada.

Velásquez Toro (2005) asevera que en los textos decimonónicos colombianos, se consideraba a la mujer como figura sagrada o como objeto de deseo. Si era «sagrada», encarnaría la transmisión del modelo religioso. Bajo este patrón, ella debía estar sujeta a la tutela de la comunidad (especialmente la masculina adulta), en calidad de esposa de Cristo y madre espiritual, o bien casada con un hombre, renunciando a los mínimos derechos que tenía en beneficio de su esposo; si era considerada «objeto de placer», se referían, en particular, a la población femenina que no pertenecía a la condición social ni étnica de los grupos dirigentes.

Bermúdez (1993), por su parte, se propone revisar todas las publicaciones contemporáneas que dieran tratamiento acerca del «bello sexo» durante el siglo XIX colombiano. Su diagnóstico es que la historia en Colombia está «rezagada» en relación a la problemática femenina; hay una indiferencia en la incorporación de la mujer a la hora de narrar los acontecimientos. La autora lo recorre en los estudios centrados en el campo de la historia y de la literatura pretendiendo evitar los análisis en torno a las «heroínas» (mujeres excepcionales). Para la autora, lo importante es dar cuenta del desenvolvimiento de las relaciones patriarcales durante el siglo XIX. No obstante, asevera que de la bibliografía consultada son contados los estudios que hacen referencia a estos años. De lo que sucede en los decenios posteriores a la independencia se conoce muy poco, pues la información no solo es escasa, sino muy general, o bien circunscrita a localidades.

Se cree que si bien ha sido necesario incorporar en las investigaciones históricas reivindicaciones en relación al sexo femenino, quizás simultáneamente el fuerte énfasis sobre la presencia/ausencia de la mujer, produjo una distracción sobre el tratamiento a fondo de las problemáticas habituales del siglo XIX: la formación del Estado, la consolidación de la Nación y el ser ciudadano en la Nueva Granada.

Para Bermúdez es fundamental abordar la historia desde una «perspectiva de género» $[s i c]$, tomando a las diferencias entre los sexos ya no desde el terreno biológico y determinista, sino desde el plano simbólico. Pero surge la interrogación: ¿cómo entender el concepto género a la luz del estudio de los pensadores del siglo XIX? ¿Cuán mezclada está la perspectiva analítica del sujeto de la escritura (Bermúdez, por ejemplo) con la de los autores decimonónicos? ¿No es anacrónico leer la primera mitad del siglo XIX colombiano desde la perspectiva de género? Por tanto, y si se insiste en utilizar este concepto, ¿̇es lo mismo hablar de género en el siglo XIX que en el XX? Si efectivamente la respuesta a esta última pregunta es negativa, entonces ¿qué reservas se deberían tomar para poder hacer de la palabra género una herramienta analítica? Algunos han entendido el concepto 
género como un modo de hacer referencia a una particularidad (género femenino o masculino), otros a una generalidad (género humano), pero en todo caso dice sobre la existencia de una diferencia.

En síntesis, se cree que no alcanza la explicación de una «sociedad o estructura patriarcal» para entender la exclusión de la mujer de la ciudadanía política y del sujeto de la nación (en construcción). Insistimos que se debe poder combinar los estudios sobre la idea de nación moderna con aquellos que tematizan las exclusiones de ella.

Por último, se ha analizado el trabajo de Dueñas Vargas (2005), quizás de los tres estudios el más aproximado a nuestra hipótesis de trabajo. La autora se centra «en las concepciones sobre cómo educar a hombres y mujeres para que se ajustaran a sus funciones de enaltecer la nación imaginada», y en las representaciones sobre el ideal femenino que «se modificaron de acuerdo con los cambios políticos, y con la variación en las representaciones respecto a lo público y lo privado» (Dueñas Vargas, 2005: 104).

Asume que la mujer, desde un lugar políticamente marginal, cumplió un papel en la conformación del mundo de la vida, «formando a los varones en las virtudes que convertían a los hombres en buenos ciudadanos», «las mujeres eran imaginadas como parte integrante de la aventura nacional» (Dueñas Vargas: 120). Sin embargo, existe una paradoja en sus premisas:

«En el hogar ellas [las mujeres] ejercían la función social que les era vedada en los escenarios políticos. La influencia de las mujeres era considerable ya que el hogar era el cruce de caminos entre lo público y lo privado. Era allí donde se forjaban en las virtudes morales a los futuros ciudadanos (Dueñas Vargas, 2005: 120).

¿Cómo es posible ejercer una función social — como lo era el formar un sujeto político, el ciudadano varón- desde un escenario no considerado «político»?

«Por un lado, la responsabilidad de las mujeres era formar a los ciudadanos en las buenas costumbres y bajo los preceptos católicos, en el recinto del hogar. El mundo de los sentimientos y la sensibilidad fue pues asignado a las mujeres. Por otro lado, los varones — liberales - haciendo rupturas y preparándose ellos, y preparando a los hijos para los debates que tenían que enfrentar en los espacios políticos. Su mundo en la nación naciente era la elaboración de las leyes y en esa dirección fueron formados (Dueñas Vargas, 2005: 113).

\section{HISTORIOGRAFÍA SIN LA FIGURA FEMENINA}

Aquellos autores que se han consultado para abordar el escenario histórico político donde se desplegaron los discursos fundacionales del concepto de Nación tanto en Argentina como en Colombia, no vislumbraron - la mayoría de ellos- el papel que jugó la mujer escrita en la construcción literaria de dicho concepto. En el presente informe se darán algunos ejemplos al respecto. 
Andrés Gordillo Restrepo (2003) tiene como objetivo principal analizar, desde las elites colombianas, la formación de la nación en torno a las producciones literarias de la primera mitad del siglo XIX producto de la tertulia «El Mosaico» (1858-1872). Es así que, si bien el autor describe y analiza las conceptos de «nación», «identidad nacional», «pueblo», etc., referidos a diversas trayectorias historiográficas y el contexto de producción de la elite intelectual; advierte que aquella elite tenía la pretensión de:

«Explicar la identidad de la nación entera, donde se ve cotejado ese imaginario de unidad, homogeneidad e indivisibilidad de la nación europea (que es una aspiración) con la realidad de una Colombia federada y mestiza (Gordillo Restrepo, 2003: 53)».

El autor atribuye como componentes de la nación al indígena, al negro y al colono mestizo de las tierras bajas [sic]. Para él, la idea de Nación no llegó, en esta literatura, «a una descripción rigurosa y homogénea» de todos estos. Asevera que el mestizo era el tipo nacional por excelencia ya que, según la elite, encarnaba e integraba la nación en sus otros componentes. Era una figura que sintetizaba a las otras.

Gordillo Restrepo revisa diversos artículos aparecidos en El Mosaico. Entre ellos hay uno que nos es de gran interés. Se titula «Antes y ahora» y estaba dedicado a las «lectorcitas del Mosaico» firmado por «un colombiano». Allí, dice Restrepo, «no sólo se criticaba el uso de la crinolina, sino que además se recriminaba la degeneración del gusto de las jovencitas, con respecto a los tiempos de la Gran Colombia y la intromisión de costumbres extranjeras, como la de tomar el té en lugar del chocolate» (Gordillo Restrepo, 2003: 51). El artículo apareció en El Mosaico, n. ${ }^{\circ} 14$, el 26 de marzo de 1859:

«Vosotras, siguiendo la corriente y el impulso de la moda, habéis querido sustituir el excelente, el sabroso, el nunca bien, como se debe, alabado chocolate, por una decocción que aquí se llama té, y que en realidad no se sabe lo que es [...] ¿En donde tenéis, bellas lectoras, el gusto y aun el buen sentido para proscribir una costumbre nacional, que hoy es bien recibida y aceptada en las mejores sociedades del viejo mundo? (citado en Gordillo Restrepo, 2003: 52)».

Se entiende que el objetivo del autor no es iluminar la figura de la mujer en aquellos artículos. No obstante, Ilama la atención el prominente lugar que esta tenía en el mencionado artículo. El imaginario en torno a la mujer, al parecer, le confería poder a ella para modificar las costumbres nacionales. La mujer pareciera encarnar la figura de «la intrusa» — si acaso quisiera modificar las costumbres_-; pero también una «excusa», para poner coto a la influencia extranjera.

Los conflictos de esos años (1831-1858) giraban en torno a la definición del sujeto político y la secularización de la sociedad. Fernán E. González se interroga:

«之Hasta qué punto los sectores denominados subalternos tenían derecho a una participación plena y autónoma en el cuerpo político? ¿Cómo modernizar una sociedad estática y patriarcal en la que se había encarnado la institución eclesiástica?» (González, 2006: 403; el énfasis es nuestro). 
Ambas preguntas nos llevan a pensar en nuestra figura. La mujer simbolizaría, por un lado, el sujeto restringido a participar en el cuerpo político, y asimismo, el imaginario en torno a la mujer como promotora del pensamiento religioso; pero también que «la emancipación de la mujer» implicaría la modernización de la sociedad.

Por último, hemos consultado en la obra de Hans Joachim König en donde encontramos un rico recorrido por los discursos acerca de la identidad, Estadonación y ciudadanía en el siglo XIX:

«Fueron sobre todo las élites políticas que lograron realizar o mejor dicho imponer su proyecto nacional y construir los imaginarios nacionales según sus visiones o sus necesidades, es decir construir la «nación〉 simbólicamente. Ante la exigencia de que las poblaciones dispersas y heterogéneas, mal unidas por lealtades locales o provinciales, se sintieran parte de las respectivas comunidades políticas, las élites no sólo se sirvieron de los símbolos cívicos clásicos, como el himno y la bandera, sino también del aparato educativo. Fueron precisamente la literatura y la historiografía oficial, con la finalidad de calmar conflictos internos y estimular identidades colectivas nacionales, las encargadas de inventar tradiciones, memorias y mitos fundacionales (König, 2005: 11)».

El autor parece sumarse a la propuesta de comprender a la nación más allá de su carácter procesual; es decir, viendo la evolución del concepto, y al mismo tiempo percibir los diferentes «proyectos nacionales» que rivalizaban entre ellos. Problematiza «el viejo concepto de nación como una «comunidad homogénea»» (König, 2005: 20) que para König es, en realidad, «una ficción que muchas veces ocultaba relaciones de dominación y exclusión». Su enfoque pretende romper con el viejo supuesto de que la creación de un Estado-nación es solamente un proceso realizado de la elite hacia el pueblo o del centro hacia la periferia. El proceso de imaginar la nación — dice el autor — es múltiple y la formación de naciones modernas es también el resultado de intensos conflictos en los cuales los grupos subordinados participaron con sus propios discursos.

Por lo tanto, la figura de la mujer, lejos de ser una figura anacrónica para el análisis de los documentos de la primera mitad del siglo XIX, es más bien una propuesta viable a través de la cual iluminaría zonas de la conformación de la nación tanto en La Nueva Granada como en el Río de la Plata.

\section{Referencias citadas}

BERMÚDEZ, S., 1993 - El «bello sexo» y la familia durante el siglo XIX en Colombia. Revisión de publicaciones sobre el tema. Revista Historia Crítica, n. ${ }^{\circ}$ 8: 34-51; Bogotá: Departamento de Historia de la Universidad de los Andes. Disponible en: http://historiacritica.uniandes.edu.co/view.php/140/index.php?id= 
Mujer y nación en el «discurso» de la intelligentzia en el amanecer de las repúblicas

BLOCH, M., 1992 - Por una historia comparada de las sociedades europeas. In: Marc Bloch. Una historia viva (G. Godoy \& E. Hourcade, eds.): 63-98; Buenos Aires: Centro Editor de América Latina.

DUEÑAS VARGAS, G., 2005 - La educación de la élites y la formación de la nación en el siglo XIX. In: IX Cátedra Anual de Historia Ernesto Restrepo Tirado. Mujer, nación, identidad y ciudadanía: siglos XIX y XX: 102-122; Bogotá: Museo Nacional de Colombia.

ECHEVERRÍA, E., 1951 [1837] - La Cautiva. In: Obras Completas de Esteban Echeverría; Buenos Aires: Ediciones Antonio Zamora.

GONZÁLEZ, F. E., 2006 - ¿Una comunidad política escindida? Guerras civiles y formación del Estado colombiano (1839-1854). In: Las revoluciones en el mundo atlántico (M. T. Calderón \& C. Thibaud, coords.): 402-431; Bogotá: Grupo Santillana, Taurus, Centro de Estudios en Historia - Universidad Externado de Colombia, Fundación Carolina Colombia.

GUILLEN, G., 2008 - El nuevo Herald, 12 de nov. de 2008.

HELIBRON, J., 2009 - Repenser la question des traditions nationales en sciences sociales. In: L'espace intellectuel en Europe. De la formation des États-nations à la mondialisation XIXe-XXe siècles (G. Sapiro, ed.): 301-317; París: La Découverte.

JURT, J., 2009 - Le champ littéraire entre le national et le transnational. In: L'espace intellectuel en Europe. De la formation des États-nations à la mondialisation XIXe-XXe siècles (G. Sapiro, ed.): 201-232; París: La Découverte.

KÖNIG, H. J., 2005 - Discursos de identidad, Estado-nación y ciudadanía en América Latina: Viejos problemas nuevos enfoques y dimensiones. Revista Historia y Sociedad, n. 11; Bogotá: Universidad Nacional de Colombia - Facultad de Ciencias Humanas y Económicas, Departamento de Historia.

NIETO GIL, J. J., 2001 [1844] - Ingermina o la hija del calamar, 216 pp.; Medellín: Fondo Editorial Universidad EAFIT.

NIETO GIL, J. J., 2010 [1842] - Rosina o la prisión del castillo de Chágres. In: http://ciruelo. uninorte.edu.co/pdf/BDC101.pdf

TORRES, M. I. de, 1995 - ¿La nación tiene cara de mujer? Mujeres y nación en el imaginario letrado del siglo XIX, 140 pp.; Montevideo: Editorial Arca.

URIBE-URAN, V., 2006 - Derecho y cultura legal durante la «Era de la Revolución» en México, Colombia y Brasil, 1750-1850: La génesis de lo público y lo privado. In: Las revoluciones en el mundo atlántico (M. T. Calderón \& C. Thibaud, coords.): 251297; Bogotá: Grupo Santillana, Taurus, Centro de Estudios en Historia - Universidad Externado de Colombia, Fundación Carolina Colombia.

VELÁSQUEZ TORO, M., 2005 - Aspectos jurídicos de la condición histórica de las mujeres en Colombia. In: IX Cátedra Anual de Historia Ernesto Restrepo Tirado. Mujer, nación, identidad y ciudadanía: siglos XIX y XX: 46-63; Bogotá: Museo Nacional de Colombia. 